\title{
$\mu$ ChemLab: twenty years of developing CBRNE detection systems with low false alarm rates
}

Joshua J. Whiting, Edward B. Myers, Ronald P. Manginell, Matthew W. Moorman, Kent Pfeifer, et al.

Joshua J. Whiting, Edward B. Myers, Ronald P. Manginell, Matthew W.

Moorman, Kent Pfeifer, John M. Anderson, Cory S. Fix, Cody Washburn, Alan Staton, Daniel Porter, Darin Graf, David R. Wheeler, John Richards,

Komandoor E. Achuythan, Michael Roukes, Robert J. Simonson, " $\mu$ ChemLab: twenty years of developing CBRNE detection systems with low false alarm rates," Proc. SPIE 11010, Chemical, Biological, Radiological, Nuclear, and Explosives (CBRNE) Sensing XX, 1101012 (17 May 2019); doi:

$10.1117 / 12.2518778$

Event: SPIE Defense + Commercial Sensing, 2019, Baltimore, Maryland, United States 


\title{
$\mu C_{h e m L a b}{ }^{\mathrm{TM}}$ - Twenty Years of Developing CBRNE Detection Systems with Low False Alarm Rates
}

Joshua J. Whiting*a , Edward B. Myers ${ }^{\mathrm{b}}$, Ronald P. Manginell ${ }^{\mathrm{a}}$, Matthew W. Moorman ${ }^{\mathrm{a}}$, Kent Pfeifer ${ }^{\mathrm{a}}$, John M. Anderson ${ }^{a}$, Cory S. Fix ${ }^{a}$, Cody Washburn ${ }^{a}$, Alan Staton ${ }^{a}$, Daniel Porter ${ }^{a}$, Darin Graf ${ }^{a}$, David R. Wheeler ${ }^{a}$, John Richards ${ }^{\mathrm{a}}$, Komandoor E. Achuythan ${ }^{\mathrm{a}}$, Michael Roukes ${ }^{\mathrm{b}}$, Robert J. Simonson ${ }^{\mathrm{a}}$

\author{
aSandia National Laboratory, 1515 Eubank Blvd SE, Albuquerque, NM, USA 87123; ' Dept. of Physics, \\ Applied Physics, and Bioengineering, California Institute of Technology, 1200 E. California Blvd., Pasadena, \\ CA USA 91125
}

\begin{abstract}
Gas Chromatography (GC) is routinely used in the laboratory to temporally separate chemical mixtures into their constituent components for improved chemical identification. This paper will provide a overview of more than twenty years of development of one-dimensional field-portable micro GC systems, highlighting key experimental results that illustrate how a reduction in false alarm rate (FAR) is achieved in real-world environments. Significantly, we will also present recent results on a micro two-dimensional GC (micro GCxGC) technology. This ultra-small system consists of microfabricated columns, NanoElectroMechanical System (NEMS) cantilever resonators for detection, and a valve-based stop-flow modulator. The separation of a 29 -component polar mixture in less than 7 seconds is demonstrated along with peak widths in the second dimension ranging from 10-60 ms. For this system, a peak capacity of just over 300 was calculated for separation in about $6 \mathrm{~s}$. This work has important implications for field detection, to drastically reduce FAR and significantly improve chemical selectivity and identification. This separation performance was demonstrated with the NEMS resonator and bench scale FID. But other detectors, suitably fast and sensitive can work as well. Recent research has shown that the identification power of GCxGC-FID can match that of GC-MS. This result indicates a path to improved size, weight, power, and performance in micro GCxGC systems outfitted with relatively non-specific, lightweight detectors. We will briefly discuss the performance of possible options, such as the pulsed discharge helium ionization detector (PDHID) and miniature correlation ion mobility spectrometer (mini-CIMS).

Keywords: Microfabricated GC, micro ChemLab, GCxGC, MEMS GC, MEMS GCxGC, False Alarm Rate, NEMS, CBRNE
\end{abstract}

\section{INTRODUCTION}

Forty years after the seminal work by Stephen Terry, Hal Jerman, and Jim Angell at Stanford University ${ }^{1}$, the field of micromachined or microelectromechanical systems (MEMS) gas chromatography (GC) is still evolving and growing. While there have been many groups in the field since then; 20 years ago Sandia National Laboratories began investing heavily in developing low size, weight and power (SWaP) MEMS technology for the use in portable systems to provide rapid, low false alarm rate (FAR) detection of chemical weapons for military and homeland security applications. ${ }^{2,3}$ The core principal is to share the burden of selectivity between the three primary analytical components (preconcentration, separation, and detection). Figure 1 shows a schematic of how this concept works, especially for the first generation of systems. The MEMS preconcentrator is coated with a thin highly polar film that rejects non-polar interferants which are exhausted to vent. The preconcentrator is rapidly heated to generate a sharp injection pulse. This injection bolus is swept by carrier gas onto the separation column where analytes are separated by boiling point typically but may also be separated by different chemical affinities, i.e. hydrogen-bond affinity, pi-pi interactions, etc. The separation column introduces the eluting compounds as discrete bands to the detector, thus reducing the number of compounds the detector sees at any given time. The detector is coated with a film that has a high Lewis acid-base interactions affinity, i.e. phosphorous and nitrogen containing compounds and to a lesser extent sulfur and oxygen. By sharing the burden of selectivity, the performance of any individual component does not have to compare to commercial benchtop instruments for the ensemble to achieve very low false alarm rates (FAR). This technology and concept were named $\mu \mathrm{ChemLab}^{\mathrm{TM}}$ and have since formed the basis for Sandia's chemical sensing technology development.

*jjwhiti@sandia.gov; phone: 1-505-845-0712 555-1234; http://www.sandia.gov/mems/sensors/index.html

Chemical, Biological, Radiological, Nuclear, and Explosives (CBRNE) Sensing XX

edited by Jason A. Guicheteau, Chris R. Howle, Proc. of SPIE Vol. 11010, 1101012

(C) 2019 SPIE · CCC code: 0277-786X/19/\$18 · doi: 10.1117/12.2518778 


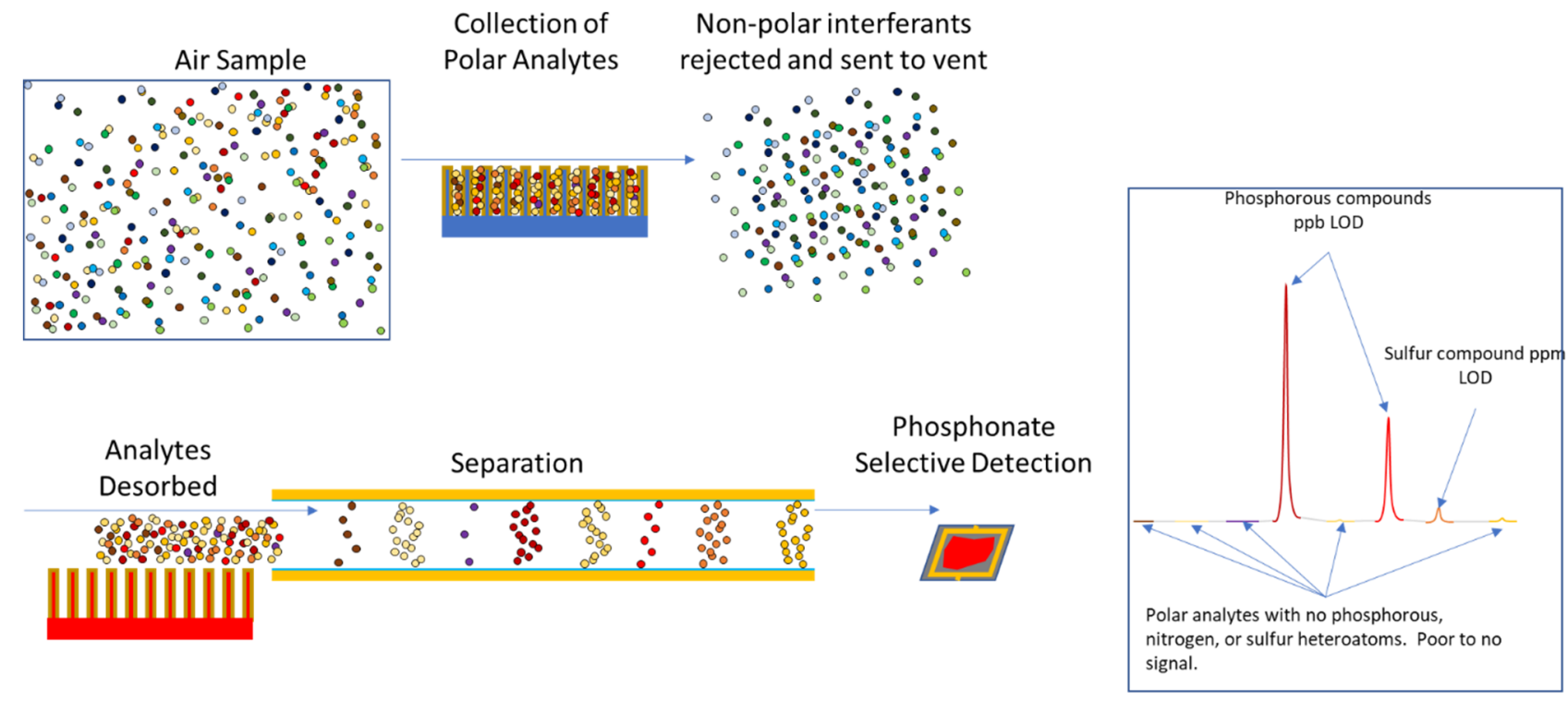

Figure 1. Schematic illustrating the shared burden of selectivity for minimizing false alarms in a $\mu$ ChemLab ${ }^{\mathrm{TM}}$ system. First, nonpolar compounds are excluded from preconcentration by a selective film. Next the polar analytes are separated on a short MEMS GC column to minimize the number of coelutions presented to the detector. Last, a phosphonate selective film on the detector minimizes detector response to anything that does not contain nitrogen, phosphorus, or sulfur.

The use of silicon microfabrication enables the reduction of size and weight of many components of analytical systems, and this has allowed the significant rise in commercial portable systems. The growth of commercial, small portable GCmass spectrometry (MS) systems has largely led this field. However, as the systems have gotten smaller, the fundamental limits of vacuum pumping capability have resulted in trade-space compromises. While the ion trap-based mass-filters see an increase in operating pressure capability as the trap volume decreases, thus enabling higher operating pressures, all the charge amplification systems still require very low pressures to operate, thus systems that choose to use higher operating pressures reducing the pumping demand are left with Faraday cup detectors similar to those used in IMS systems. This results in either optimizing for SWaP at the expense of sensitivity or optimizing for sensitivity at the expense of SWaP. This can be seen in two of the popular systems on the market: the portable the Perkin Elmer/Torion system, which sacrifices SWaP by adding larger batteries and a turbo pump to achieve greater sensitivity and the 908 Devices Inc. handheld M908 which uses smaller batteries and a scroll pump to achieve greater SWaP at the expense of sensitivity. Sandia has developed microfabricated ion traps, but the $\mu$ ChemLab ${ }^{\mathrm{TM}}$ programs have focused on systems where decreasing SWaP results in a lower loss of analytical power. In an effort to quantify these approaches, Analytical Power $\left(A_{p}\right)$ has been defined as the product of column resolution $(\mathrm{N})$, detector channels (n), and detector sensitivity (D) as shown in equation 1.

$$
\mathrm{A}_{\mathrm{p}}=\mathrm{N} \cdot \mathrm{n} \cdot \mathrm{D}
$$

Figure 2 shows estimates of the SWaP and calculated $\mathrm{A}_{\mathrm{p}}$ of several commercial systems and Sandia's first and second generation $\mu \mathrm{ChemLab}{ }^{\mathrm{TM}}$ systems. The first generation $\mu \mathrm{ChemLab}^{\mathrm{TM}}$ system offered nearly 7 orders of magnitude decrease in SWaP in exchange for 4 orders of magnitude decrease in $\mathrm{A}_{\mathrm{p}}$ relative to the benchtop GC-MS, long considered to be the "gold standard" of analytical chemistry. To achieve this, the first $\mu$ ChemLab ${ }^{\text {TM }}$ team and subsequent teams since realized the SWaP impediment of the pumping requirements for portable MS systems, and focused on approaches where microfabrication would provide improvements in performance over macro machined components. These included the microfabricated GC, preconcentrator (PC) and resonator-based detectors. The use of a micromachined PC enables lowerpower, faster heating which results in a narrower injection plug into the $\mathrm{GC}$ - this enhances the resolution of the separation at a fraction of the power demand. Modeling predicted microfabrication of GC columns would offer improved efficiency per unit length relative to commercial columns, with again the additional benefit of lower-power, faster heating enabling faster temperature ramps enabling faster analysis times. Resonators have a natural resonance frequency that is inversely proportional to their mass $(\mathrm{m})$. When coated with a sorptive film, resonators will respond to the change in mass $(\Delta \mathrm{m})$ 
from the added mass as analytes sorb into/onto the film with a decreasing in the resonance frequency. This response is proportional to the change in mass relative to its mass $(\Delta \mathrm{m} / \mathrm{m})$. As the mass of the resonator decreases the effective sensitivity increases. In addition, the as the resonator decreases in size the volume of the detector decreases, improving separation resolution and response time. Sandia has also focused on using chemical selectivity to minimize the number of interferants introduced into a system and the detectability of interferants by the system. The result was a low SWaP system that punches well above its weight class for a specific targeted analysis. The tradeoff is the versatility of the system, an area where hand-held and portable GC-MS systems have a significant advantage over this first generation $\mu \mathrm{ChemLab}^{\mathrm{TM}}$ system.

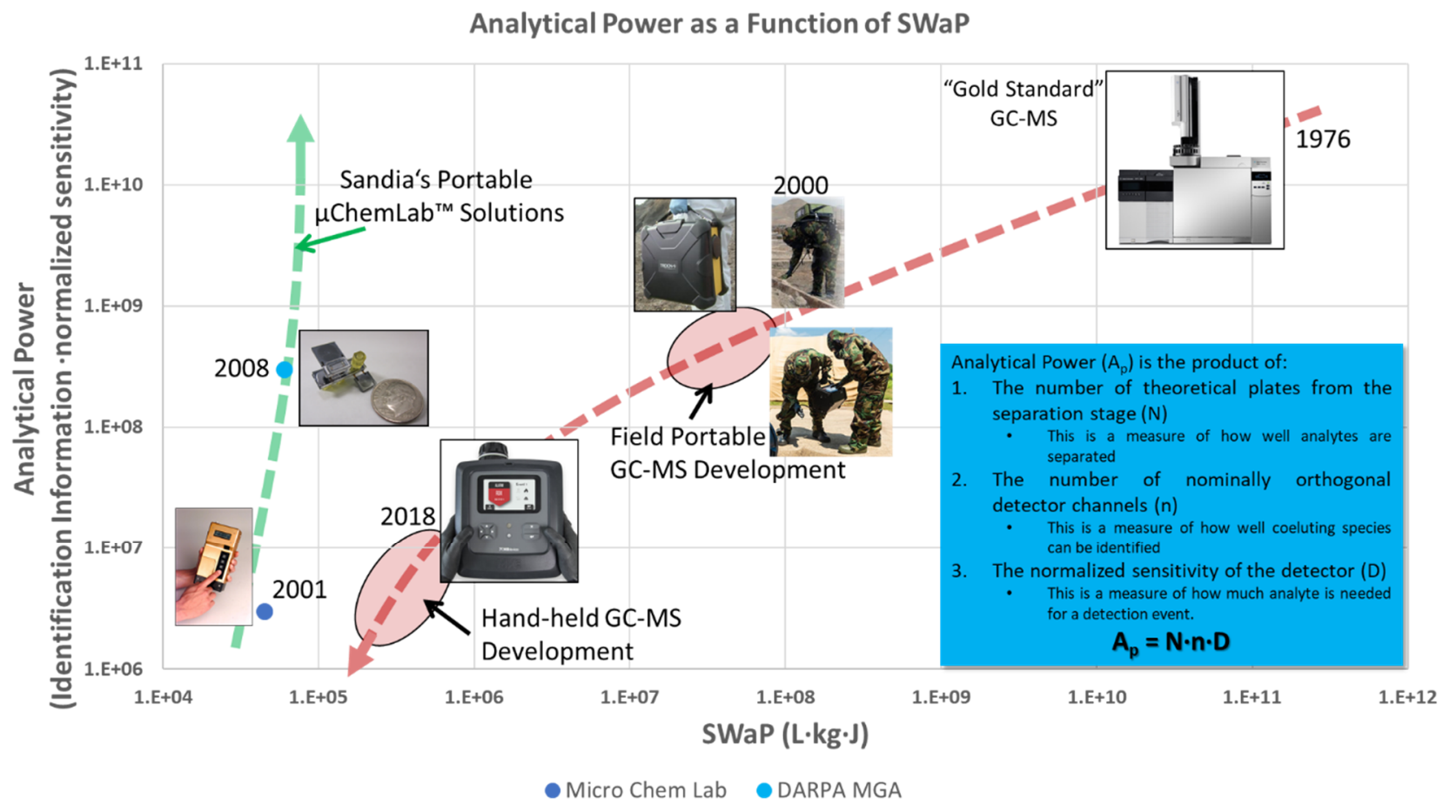

Figure 2. Comparison of Analytical Power as a function of system SWaP comparing commercial GC-MS systems with $\mu$ ChemLab ${ }^{\mathrm{TM}}$ systems

\section{FIRST GENERATION $\boldsymbol{\mu C H E M L A B}{ }^{\mathrm{TM}}$}

This targeted low SWaP first generation $\mu \mathrm{ChemLab}^{\mathrm{TM}}$ system was highly amenable to monitoring applications. With low power demands, a battery-operated unit could be installed nearly anywhere including unmanned aerial vehicles (UAVs) and operate for hours or days, and a system using wall power could be installed and operated for months to years. Examples of this are shown in Figure 3. On the top is the PRO-ACT system which was installed in the Boston subway for continuously monitoring the presence of chemical warfare agents (CWAs). The system performed over $450,000^{4}$ analyses with autonomous operation and calibration, with no false alarms and no false negatives. Below this, in Figure 3, is the UAV mounted SnifferSTAR ${ }^{5}$ system which decreased the SWaP even more by eliminating the separation stage and pumps. The air impinging on the wing of the UAV was passed over the selective preconcentrator and at regular intervals the preconcentrator was heated and the captured analytes were desorbed onto the SAW detector. In this way, CWA classes could be identified and large areas (such as cities or battlefields) could be monitored in near real-time. 


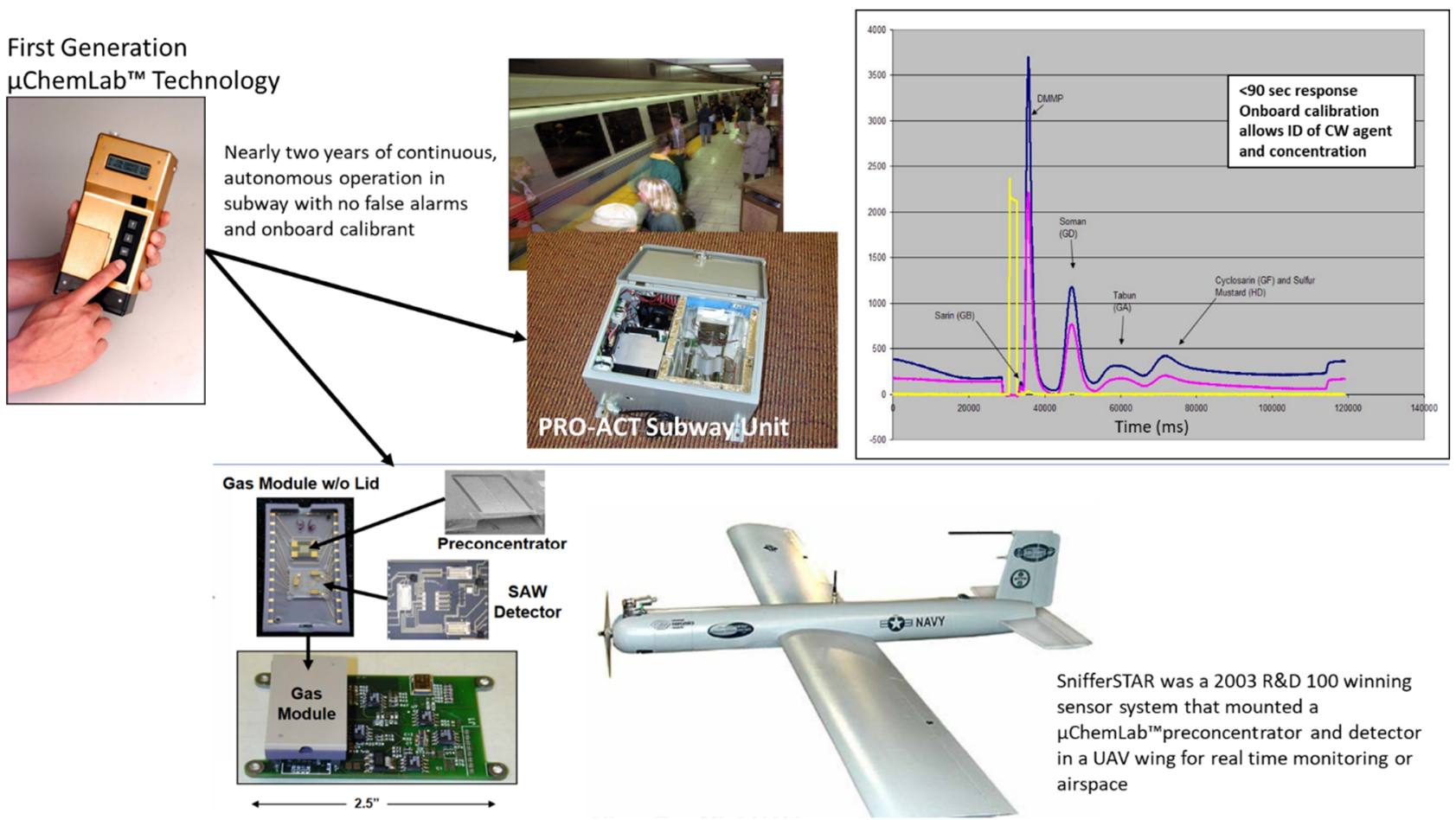

Figure 3. Fielded first generation $\mu$ ChemLab ${ }^{\mathrm{TM}}$ based systems. At top the PRO-ACT Subway system monitored air in a subway station for 22 months with no false alarm rates. A representative chromatogram from such a system showing the separation of nerve agents and DMMP calibrant in 90s. At bottom the SnifferSTAR, a 2003 R\&D 100 winner, utilizing the selective preconcentrator with selective detection for real time UAV-based atmospheric monitoring.

\section{SECOND GENERATION $\mu$ CHEMLAB ${ }^{T M}$}

Over time, advances in micromachining and new ideas and technology emerged to advance the chemical sensing mission at Sandia. These included internally funded developments such as the monolithically integrated ultra-low SWaP $\mu \mathrm{GC}$ system $^{6,7}$ and the DARPA/DTRA funded development of the microfabricated ultra-low SWaP, stop-flow comprehensive two-dimensional gas chromatograph $(\mu \mathrm{GCxGC})^{8,9}$. In addition, developments in other areas at Sandia, such as miniaturized positive and negative mode ion mobility spectrometers (IMS) ${ }^{10,11}$ for the detection of CWAs and explosives; pulsed discharge helium ionization detector (PDHID) ${ }^{12,13}$ for universal detection of $\mathrm{VOCs}^{14}$ and permanent gases; and microfabricated nitrogen-phosphorus detector $(\mu \mathrm{NPD})$ for the detection of organophosphorus compounds ${ }^{15}$ have increased the options for detectors.

\subsection{Smart Preconcentrator (SMART PC)}

The second-generation Sandia SMART PC ${ }^{6,7}$ is an ultra-low power Lorentz force induced resonator shown in Figure 4. It may be coated with either a selective film such as Sandia's base catalyzed sol-gel or a more generalized sorbent film such as PDMS. As analytes are collected on the film, the mass of the resonator increases, causing a drop in the resonance frequency. A base catalyzed sol-gel coated SMART PC enables real time monitoring of potential threats with very low power draws, until a potential threat is detected. This enables putting the GC in a sleep mode until sufficient analyte is collected to indicate a detection event is likely, and an alert can be given simultaneous with further analysis with the GC system to identify the threat. A drawing, optical image of the device, an SEM side view of the SMART PC with the device artificially color-enhanced in blue, and the SMART PC integrated into a Gen $1 \mu \mathrm{ChemLab}^{\mathrm{TM}}$ system are shown on the left of Figure 4. On the right is a plot of analyte concentration in ppm as a function of detection time. This shows how the device can autonomously adjust its sampling time dependent on concentration, i.e. at low-to-zero concentrations, the system will be sit in monitoring mode for minutes to hours to days, until sufficient analyte was is collected to trigger a 
response. Alternatively, in at relatively high concentrations (hundreds of ppb), the SMART PC will trigger in milliseconds to seconds enabling efficient and effective monitoring with minimal SWaP.
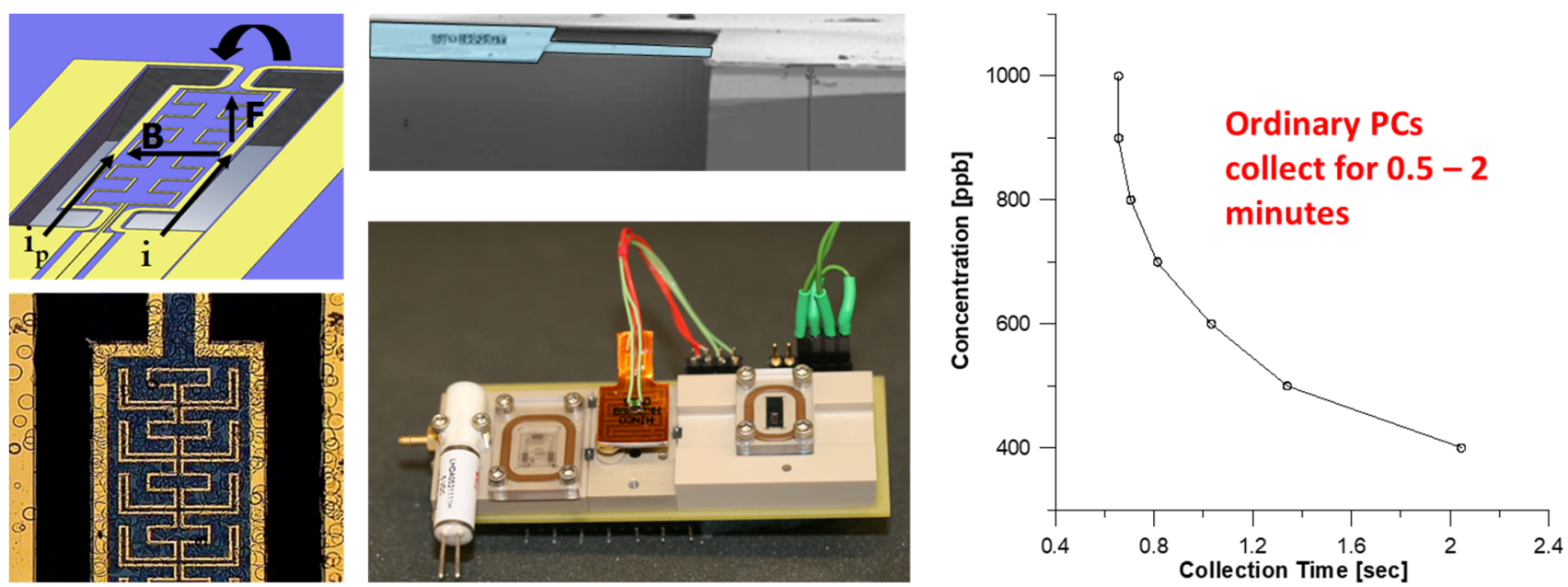

Figure 4. (Top left) Schematic showing the ultra-low power Lorentz force-based modulation method of the SMART PC. Current moving through metal loops in a magnetic field induce a force that sets the paddle structure into resonance. (Top middle) Color-modified SEM image showing the suspended paddle structure. (Bottom left) Close-up of a SMART PC paddle coated with a sol-gel collection film. (Bottom middle) Fixturing of the second generation $\mu$ ChemLab ${ }^{\mathrm{TM}}$ system showing a SMART PC collector, a $\mu \mathrm{GC}$ column, and a surface acoustic wave detector. (Right) Plotted data showing the response time for a 3:1 signal to noise ratio as a function of nerve agent simulant concentration. This demonstrates that as the concentration of analyte increases, the time to an alarm trigger decreases.

\subsection{Correlated Ion Mobility Spectrometry (CIMS)}

Correlated Ion Mobility Spectrometry (CIMS) is a Sandia-devised method to enhance the resolution and sensitivity of IMS by using a 13-bit Barker code to impart information in the gate drive signal of an IMS ${ }^{11}$. In a traditional IMS the signal enhancement achieved from averaging, assuming the signal stays constant over the $N$ sweeps, is a $\sqrt{N}$ increase in signalto-noise, but in transient systems such as ene the monitoring of GC elution peaks, the signal-to-noise enhancement is limited. Signal from the detector is a convolution of the gate drive function and the drift tube function. By imparting a known drive function, the drift function can be deconvolved resulting in an enhanced signal. One such drive function is the 13-bit Barker code used by Sandia. Figure 5 below shows at the top the Barker Code, the resulting gate drive signal, the individual ion swarms introduced into the drift tube, and the sum of the ion swarms, all as a function of time. In the bottom row on the left is signal from a $2 \mathrm{ng}$ sample of an explosive (PETN) without correlation, and on the right with correlation. An enhancement of the signal-to-noise ratio by $>15 \mathrm{X}$ and the IMS peak resolution by $>5 \mathrm{X}$, is achieved enabling smaller, lower power drift tubes, while achieving the same or better performance as systems with greater SWaP. 

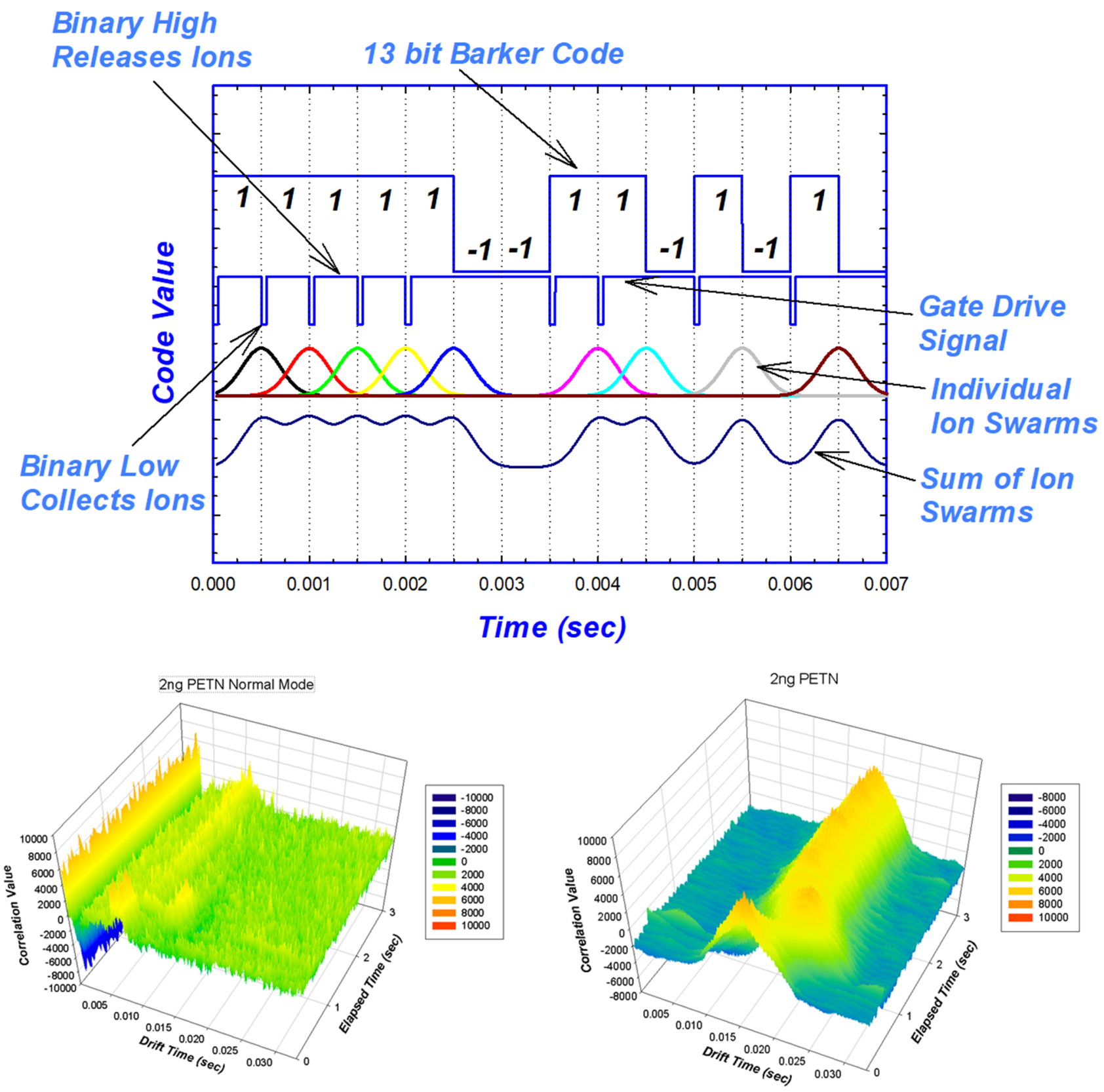

Figure 5. (Top) Figure demonstrating the realization of a 13-bit Barker Code, the resulting gate drive signal, the individual ion swarms introduced into the drift tube, and the sum of the ion swarms all as a function of time. (Bottom) A representative detection event of $2 \mathrm{ng}$ of the explosive (PETN) without correlation, and on the right with correlation which increases peak resolution and increases the signal to noise ratio.

While traditional IMS drift tubes use stacks of insulators and metal electrodes to build, the low SWaP Sandia IMS is achieved by a rolled low-temperature co-fired ceramic (LTCC) that integrates the field electrodes, heating element and temperature sensing elements into a single tube. The smooth rolled tube eliminates crevices and imperfections in the tube walls that can result in loss of analyte or tailing of analyte packets, reducing the IMS resolution and sensitivity. The LTCC drift tube eliminates these problems enabling a shorter drift tube to achieve similar or better performance. Figure 6 shows a photograph of a Sandia-designed IMS system consisting of the LTCC drift tube with integrated electrodes and heaters 
and a $20 \mu \mathrm{Ci}$ Americium-241 ionization source (an ionization source similar to those found in many smoke detectors and not subject to NRC transport restrictions as a result). The total volume of the IMS is less than the volume of a soda can. By coupling this low SWaP design with the correlation IMS gate operation and backend deconvolution, the result is a system with a significantly better performance than other IMS systems of similar size as shown by the $500 \mathrm{pg}$ detection of common explosives (bottom right).

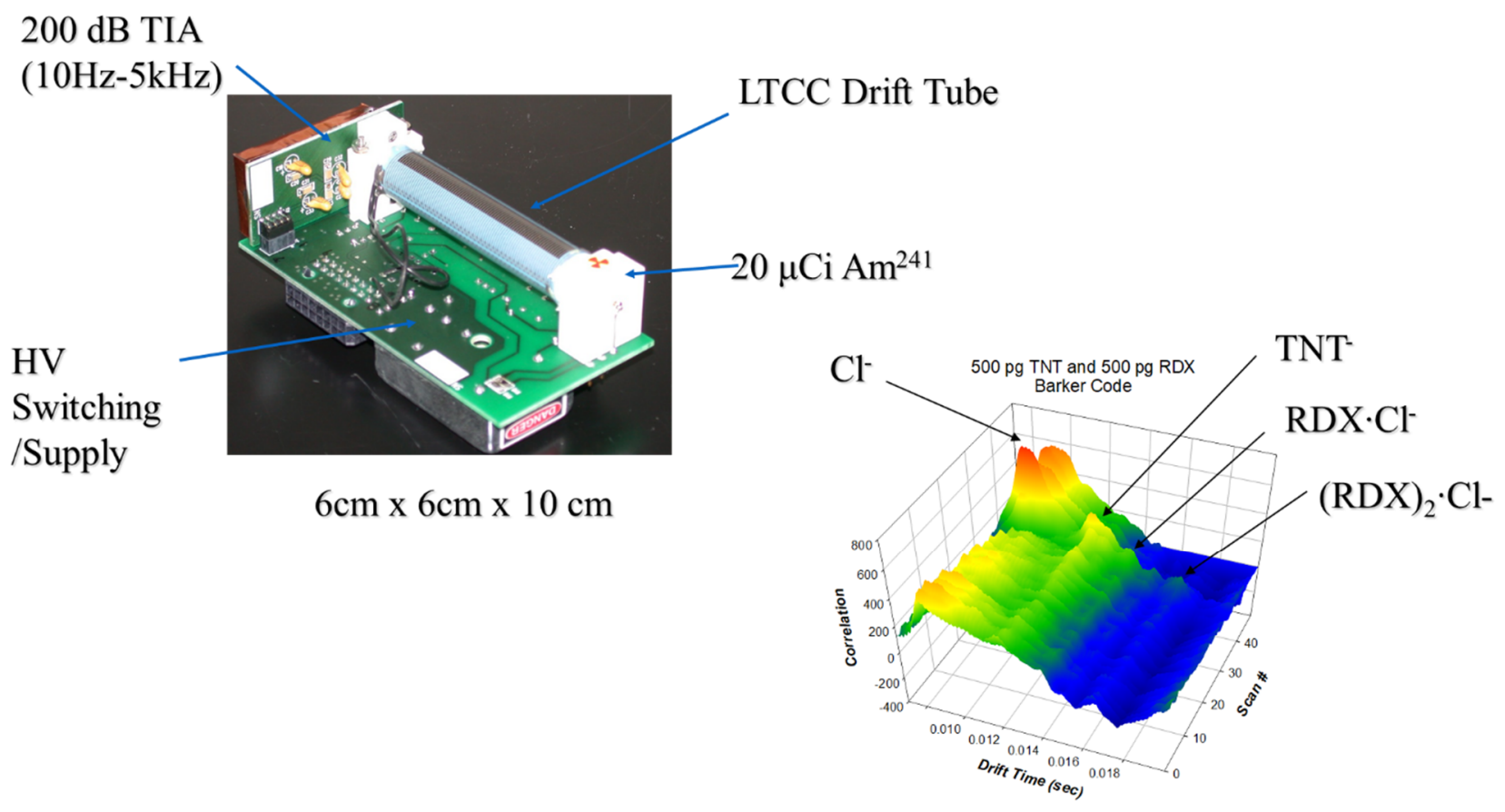

Figure 6. (Top left) Photo of a Sandia designed IMS system consisting of a rolled, low-temperature co-fired ceramic (LTCC) drift tube with integrated electrodes and heaters, a $20 \mu \mathrm{Ci}$ americium 241 ionization source, and detection electronics. (Bottom right) Simultaneous detection of $500 \mathrm{pg}$ of TNT and RDX on the CIMS detector.

\subsection{Microfabricated Stop-Flow Comprehensive Two-Dimensional Gas Chromatography ( $\mu \mathrm{GCxGC)}$}

The GC $\times$ GC modulator may in general be considered as having a two-state duty cycle. The purpose of the modulator during one state of this cycle is to stop a given elution band's migration at the end of the first column, long enough for an entire second column separation to occur. In the second state of the duty cycle, the modulator must then promote band migration at the end of the first column, resulting in injection of the next sample aliquot onto the second column. The band migration velocity of the chemical component $i$ at the modulator, $u_{i m}$, is given by equation (2), where $u_{m}$ is the linear

$$
u_{i m}=\frac{u_{m}}{\left(k_{i m}+1\right)}
$$

carrier gas velocity at the modulator, and $k_{i m}$ is the retention factor of component $i$ at the modulator. It is clear from this equation that the band migration velocity of component $i$ can be driven toward zero by either increasing the retention factor toward infinity or by decreasing the linear carrier gas velocity to zero. Traditional thermal modulators act by modulating $k_{i m}$; however, for very volatile species, including permanent gases, $k$ typically cannot be increased sufficiently to trap the analyte on the modulator. Conversely, for chemical components of very low volatility, $k$ can often not be decreased enough to get sharp injections onto the second column. Both volatility extremes result in losses of resolving power. By modulating the carrier gas velocity, $u_{m}$, instead of the retention factor, the modulation efficiency is independent of the component volatility, thus allowing a wider range of analytes to be modulated. 
By connecting the junction point between two columns to the inlet (I) of the pressure of the first column $\left(\mathrm{C}_{1}\right)$ with a valve (V), as shown in the top left of Figure 7, the valve can be used to modulate $u_{m i}$. When the valve is open, the junction point pressure is essentially set equal to the inlet pressure, effectively stopping the flow on the first column and increasing the flow on the second column. When the valve is closed a small amount of material elutes from the first column onto the second column. The flow rate at the junction point during this elution is relatively slow, because the total flow impedance between the inlet pressure source and the system outlet is the sum of the impedances of the first and second GC columns. When the valve opens, this material is separated on the second column. The flow rate on the second column during this condition is increased, due to the decrease in total gas flow impedance achieved by bypassing the first GC column. By modulating this valve position at a known duty cycle and by ensuring that all of the material introduced into the second column elutes and is detected before the next injection onto the second column elutes from the system, the retention time from the primary column is preserved and the retention time on the second column is preserved. The resulting chromatogram is then typically plotted as a retention plane, such as that shown in the top right of Figure 7. This 2D chromatogram is the result of the separation of a 30-component mixture of CWA surrogates and common interferants, listed in Table 1, on a pair of microfabricated columns shown in the bottom left of Figure 7 . The
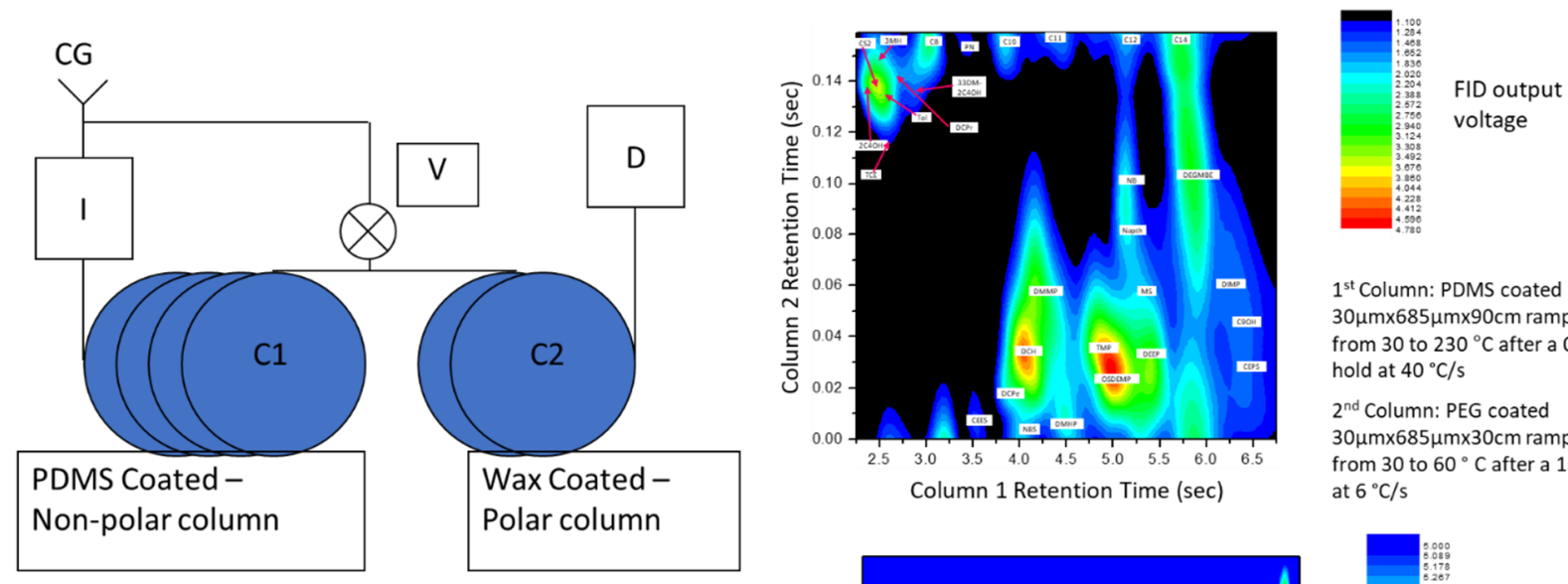

$1^{\text {st }}$ Column: PDMS coated $30 \mu \mathrm{m} \times 685 \mu \mathrm{m} \times 90 \mathrm{~cm}$ ramped from 30 to $230^{\circ} \mathrm{C}$ after a $0.5 \mathrm{~s}$ hold at $40^{\circ} \mathrm{C} / \mathrm{s}$

\section{$2^{\text {nd }}$ Column: PEG coated} $30 \mu \mathrm{m} \times 685 \mu \mathrm{m} \times 30 \mathrm{~cm}$ ramped from 30 to $60^{\circ} \mathrm{C}$ after a $1 \mathrm{~s}$ hold Column 1 Retention Time (sec)
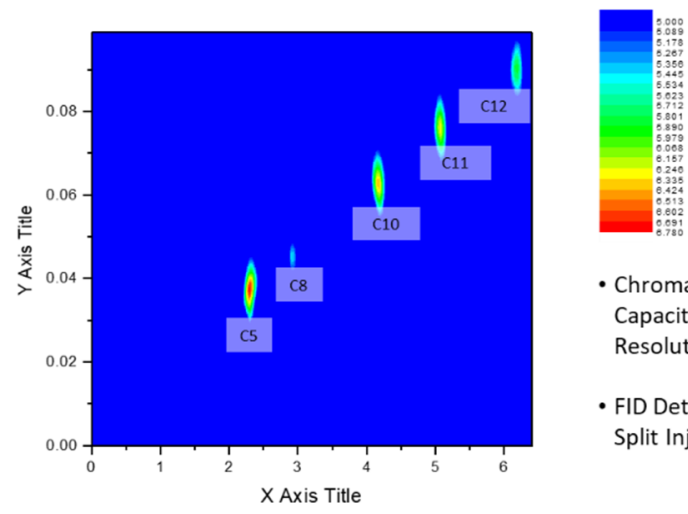

- Chromatographic Peak Capacity calculated for Resolution of 1: $\mathrm{Cp}=307$

- FID Detection, Standard Split Injection

Figure 7. (Top left) Schematic of the Sandia developed ultra-low SWaP, ultra-high-speed, stop-flow comprehensive twodimensional gas chromatograph (GCxGC) that uses pneumatic switching from an inlet pressure (I), through a very small, lowpower valve $(\mathrm{V})$ to modulate sample from the first to the second column at a known frequency. When the valve is closed the pressure at the inlet pushes flow through the first column (C1) loading effluent into the second column (C2), but when the valve opens the pressure at the junction point between the columns equilibrates with the inlet pressure effectively stopping the flow on the first column and rapidly increasing the flow on the second column. (Bottom left) Images of the first and second $\mu \mathrm{GC}$ columns used in the realization of the system. (Top right) Separation of a 30-component mixture of CWA simulants and common interferants separated in less than 7 seconds. (Bottom right) A chromatographic peak capacity of over 300 is calculated from this 7 second separation. 
Table 1. List of analytes used in testing, labels, boiling points, CAS no. and mass injected on column for Fig.7

\begin{tabular}{|c|c|c|c|c|}
\hline Analyte & Label & $\begin{array}{c}\text { Boiling } \\
\text { point }\left({ }^{\circ} \mathrm{C}\right)\end{array}$ & CAS no. & $\begin{array}{c}\text { Mass for Fig. } 7 \\
\text { (g) }\end{array}$ \\
\hline Pentane & $\mathrm{C} 5$ & 36 & $109-66-0$ & - \\
\hline Dichloromethane & DCM & 39.8 & $75-09-2$ & - \\
\hline Carbon disulfide & $\mathrm{CS} 2$ & 46 & $75-15-0$ & $4 \times 10^{-6}$ \\
\hline Trichloroethylene & TCE & 87.2 & $79-01-6$ & $5 \times 10^{-10}$ \\
\hline Chloroethylphenylsulfide & CEPS & 90 & $5535-49-9$ & $4 \times 10^{-10}$ \\
\hline 3-Methylhexane & $3 \mathrm{MH}$ & 90.7 & $589-34-4$ & $2 \times 10^{-10}$ \\
\hline 2-Butanol & $2 \mathrm{C} 4 \mathrm{OH}$ & 94 & $78-83-1$ & $3 \times 10^{-10}$ \\
\hline Dichloropropane & DCPr & 96 & $78-87-5$ & $4 \times 10^{-10}$ \\
\hline iso-Octane & iOct & 99 & $540-84-1$ & - \\
\hline Toluene & TOL & 111 & $108-88-3$ & $3 \times 10^{-10}$ \\
\hline 3,3-Dimethyl-2-butanol & $33 \mathrm{DM} 2 \mathrm{C} 4 \mathrm{OH}$ & 120 & $464-07-3$ & $3 \times 10^{-10}$ \\
\hline Octane & $\mathrm{C} 8$ & 126.4 & $111-65-9$ & - \\
\hline Chlorobenzene & CB & 131 & $108-90-7$ & $3 \times 10^{-10}$ \\
\hline Pentanitrile & PN & 140 & $110-59-8$ & $2 \times 10^{-10}$ \\
\hline Chloroethylethylsulfide & CEES & 156 & $693-07-2$ & $3 \times 10^{-10}$ \\
\hline 2-Heptanol & $\mathrm{C} 7 \mathrm{OH}$ & 161 & $543-49-7$ & - \\
\hline Dichlorobutane & DCB & 162 & $110-56-5$ & - \\
\hline Dimethyl hydrogen phosphonate ${ }^{\underline{a}}$ & DMHP & 170.5 & $868-85-9$ & $7 \times 10^{-9}$ \\
\hline Decane & $\mathrm{C} 10$ & 174 & $124-18-5$ & $2 \times 10^{-10}$ \\
\hline Dimethyl methylphosphonate $\underline{\underline{a}}$ & DMMP & 181 & $756-79-6$ & $7 \times 10^{-9}$ \\
\hline Dichloropentane & DCPe & 182 & $628-76-2$ & $3 \times 10^{-10}$ \\
\hline$n$-Butylsulfide & NBS & 188 & $544-40-1$ & $3 \times 10^{-10}$ \\
\hline Diisopropyl methylphosphonate $\underline{\underline{b}}$ & DIMP & 189 & $1445-75-6$ & $7 \times 10^{-9}$ \\
\hline Diethyl methylphosphonate & DEMP & 194 & $683-08-9$ & - \\
\hline Octyl alcohol & $\mathrm{C} 8 \mathrm{OH}$ & 194.5 & $111-87-5$ & - \\
\hline Undecane & C11 & 196 & $1120-21-4$ & $2 \times 10^{-10}$ \\
\hline Trimethylphosphate & TMP & 197 & $512-56-1$ & $7 \times 10^{-9}$ \\
\hline Diethyl ethylphosphonate & DEEP & 198 & $78-38-6$ & $6 \times 10^{-9}$ \\
\hline Dichlorohexane & $\mathrm{DCH}$ & 208 & $2163-00-0$ & $3 \times 10^{-10}$ \\
\hline Nitrobenzene & NB & 211 & $98-95-3$ & $4 \times 10^{-10}$ \\
\hline Nonyl alcohol & $\mathrm{C} 9 \mathrm{OH}$ & 214 & $143-08-8$ & $3 \times 10^{-10}$ \\
\hline Triethylphosphate & TEP & 215 & $78-40-0$ & - \\
\hline Dodecane & $\mathrm{C} 12$ & 216 & $112-40-3$ & $2 \times 10^{-10}$ \\
\hline Napthalene & Napth & 218 & $91-20-3$ & $4 \times 10^{-9}$ \\
\hline Methyl salicylate $\underline{\underline{a}}$ & MS & 222 & $8024-54-2$ & $4 \times 10^{-10}$ \\
\hline$O, S$-Diethyl methylphosphonate ${ }^{\underline{b}}$ & OSDEMP & 223 & $2511-10-6$ & $7 \times 10^{-9}$ \\
\hline Diethyleneglycol monobutylether & DEGMBE & 230 & $112-34-5$ & $6 \times 10^{-9}$ \\
\hline Tetradecane & C14 & 253 & $629-59-4$ & $2 \times 10^{-10}$ \\
\hline Diisobutyl methylphosphonate $\underline{\underline{b}}$ & DIBMP & 254 & $7242-56-0$ & - \\
\hline$O, S$-Diisobutyl methylphosphonate $\underline{\underline{b}}$ & OSDIBMP & 273.2 & $100860-55-7$ & - \\
\hline Tributylphosphate & TBP & 289 & $126-73-8$ & - \\
\hline Dipinacolyl methylphosphonate $\underline{\underline{b}}$ & DPACMP & 306.2 & $7040-58-6$ & - \\
\hline
\end{tabular}

The first column consists of a channel $30 \mu \mathrm{m}$ wide by $650 \mu \mathrm{m}$ deep and $90 \mathrm{~cm}$ in length, functionalized with a non-polar polydimethylsiloxane PDMS) stationary phase. The second column consists of a channel $30 \mu \mathrm{m}$ wide by $650 \mu \mathrm{m}$ deep and $30 \mathrm{~cm}$ in length, functionalized with a polar polyethylene glycol (PEG) stationary phase. The resulting retention plane has analytes separated as a function of boiling point along the $\mathrm{x}$ axis (increasing boiling point results in a later elution time 
- left to right), and as a function of hydrogen bonding along the y axis (increasing hydrogen bonding polarity results in a later elution time - bottom to top). Using this pair of columns and optimizing conditions for maximum resolution a peak capacity $>300$ was generated in less than $7 \mathrm{~s}$ of total analysis time.

The microfabricated $\mathrm{GC} \times \mathrm{GC}$ system shown in figure 7 was integrated with the Caltech NEMS resonator, ${ }^{8,9,16}$ as shown in the top of Figure 8. The middle row left of Figure 8 shows the isothermal separation of six components, four known targets and two unknown contaminants detected by the NEMS resonator. There was no apparent additional peak broadening from the use of the NEMS resonators. The middle row right of Figure 8 shows individual second dimension slices A, B, and C corresponding to the similarly labeled lines in the middle row left chromatogram. The slices show second dimension peaks as narrow as $8 \mathrm{~ms}$ wide (slice A) with no discernable peak distortion due to under-sampling by the NEMS resonators. The Bottom right image of Figure 8 shows an integrated GCxGC system with the preconcentrator on top a 37-mixed passive and active MEMS valve manifold for gas handling and modulation, which forms the center body of the system with the GC columns attached on the edges. The detector (not shown) could be any of the microfabricated detectors mentioned in this paper.
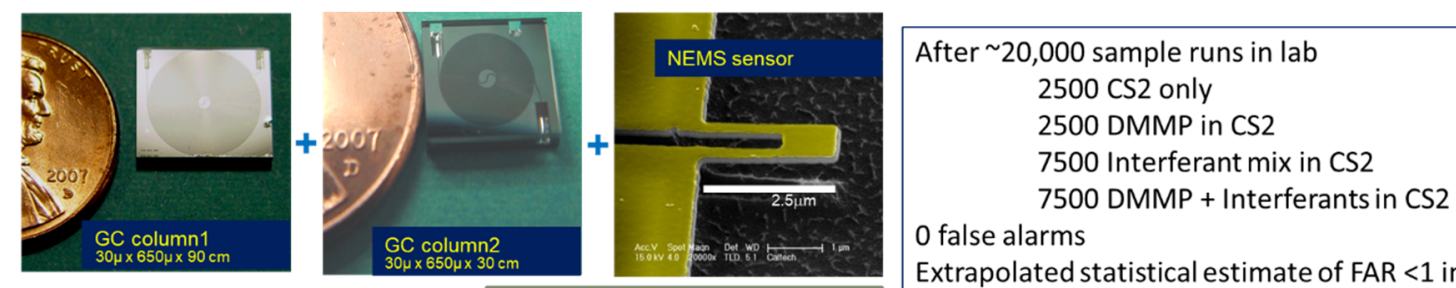

0 false alarms

Extrapolated statistical estimate of FAR $<1$ in 700 Billion
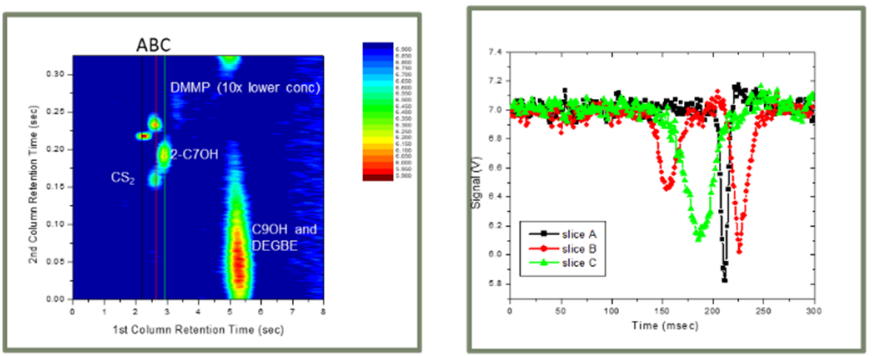
analysis

Microfabricated High Speed GCxGC + Sample Injector

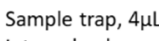
internal volume Multiple valve module sample injection and GCxGC modulation

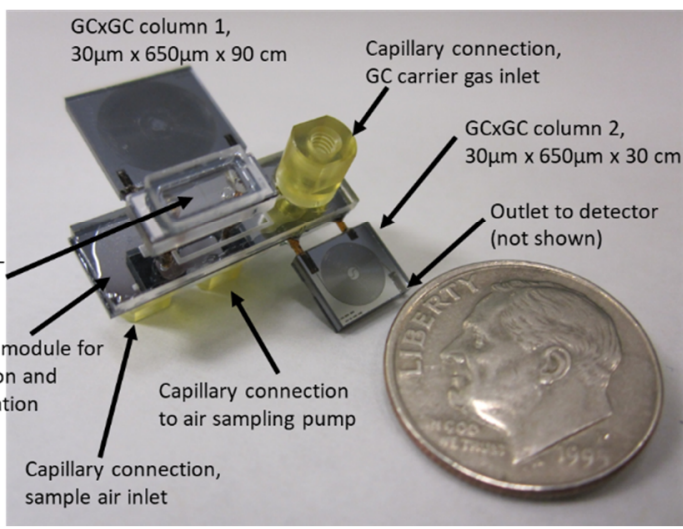

Figure 8. (Top left) Integration of the stop-flow GCxC system shown in figure 7 with a NEMS resonator developed by the Roukes' group at Caltech and operated continuously for $\sim 20,000$ cycles over the period of just over a month. (Middle left) Separation of a CWA simulant (DMMP) from common interferants not rejected by the Sandia preconcentrator coating. (Middle) Individual slices from chromatogram in (Middle left) showing the second-dimension peak widths including an $8 \mathrm{~ms}$ wide peak with no apparent distortion due to under sampling in trace A. (Top right) Analysis of the 20,000 cycles showing no false alarm events, and thru statistical extrapolation an estimated FAR of $>1$ in 700 Billion was calculated for these specific test conditions. (Bottom right) An integrated GCxGC system with the preconcentrator on top a 37-mixed passive and active MEMS valve manifold for gas handling and modulation forms the center body of the system with the GC columns attached on the edges.

Figure 9. Shows data from one subset ( $~ 1000$ DMMP-only training set) of a large ( $>20000$ analyses over 40 days) test for the evaluation of the stop-flow modulator and NEMS stability and determination of a FAR for DMMP and a set of interferants. The NEMS resonators and columns were run isothermally at $82{ }^{\circ} \mathrm{C}$ to minimize variation due to thermal cycling of the microfabricated columns. The vertical axis of the plot shown in Figure 9 is the run number in this subset, i.e. loop 14 corresponds to the 14th run of DMMP only. These loops were dispersed regularly throughout the entire 20000 
set (the other sets are described in the top right of Figure 8. The horizontal axis of the plot shown in Figure 9 is the data point in an individual run with data collected at $1 \mathrm{kHz}$, i.e. sample number 10000 corresponds to the data point for the frequency of the resonator at 10000 seconds into the run. The amplitude of the plot shown in Figure 9 corresponds to the amplitude of the data point in the loop number on the vertical axis and the sample number on the horizontal axis. Therefore, the vertical stripes at $\sim 2600,2900,3200$, and $3500 \mathrm{~ms}$ correspond to peak apexes for CS2 and DMMP. The perpendicularity of the lines corresponds to the time stability of the microfabricated $\mathrm{GC} \times \mathrm{GC}$ modulator. The signal strength stability is denoted by the uniformity of the color of each stripe and the background. This signal stability corresponds to a convolution of several factors - the stability of the sensor response, the stability of the sample introduction method, and the stability of the sample concentration. Even with these confounding factors the sensor response appears very stable over the course of 40 days of continuous operation.

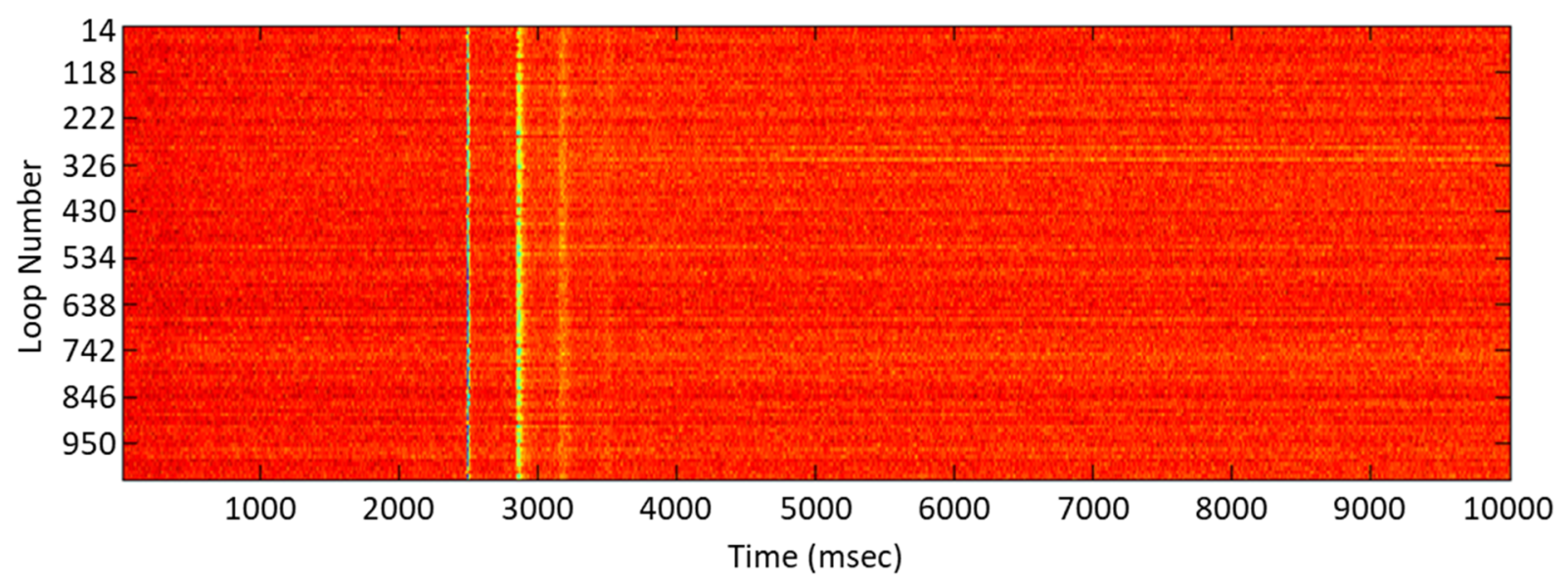

Figure 9. (Top left) Integration of the stop-flow $\mathrm{GCxC}$ system shown in figure 7 with a NEMS resonator developed by the Roukes' group at Caltech and operated continuously for $\sim 20,000$ cycles over the period of just over a month. (Middle left) Separation of a CWA simulant (DMMP) from common interferants not rejected by the Sandia preconcentrator coating. (Middle) Individual slices from chromatogram in (Middle left) showing the second-dimension peak widths including an $8 \mathrm{~ms}$ wide peak with no apparent distortion due to under sampling in trace A. (Top right) Analysis of the 20,000 cycles showing no false alarm events, and thru statistical extrapolation an estimated FAR of $>1$ in 700 Billion was calculated for these specific test conditions. (Bottom right) An integrated GCxGC system with the preconcentrator on top a 37-mixed passive and active MEMS valve manifold for gas handling and modulation forms the center body of the system with the GC columns attached on the edges.

Using an automated detection algorithm to identify the presence of DMMP or not for the 20,000 run data set with a probability of detection threshold $>0.95$ There were no empirical false detection events. Using a statistical extrapolation of the data using a gaussian fit of the response distributions and theoretical values of Pd and Pfa of 0.996 and 0.950 respectively, a probability of a false alarm for the interferents tested was $1.4 \times 10^{-12}$ which correlates to a FAR $>1$ in 700 billion analysis.

\section{CONCLUSIONS}

The $\mu$ ChemLab ${ }^{\mathrm{TM}}$ programs at Sandia have had 20 years of success developing low-SWaP, highly sensitive CBRNE detection systems with very low false alarm rates through intelligent design, distributing the selectivity burden across all components, and microfabricated components. Sandia has worked to maintain the low FAR standards we have set in the past while broadening application space by new innovations and enhancing the performance of core components to increase the analytical power of the systems while maintaining and, for some applications, reducing the low SWaP of our past. A major thrust of the $\mu \mathrm{ChemLab}^{\mathrm{TM}}$ program was system portability for field analysis of chemical, biological, radiological, nuclear and explosive (CBRNE) volatiles. This was accomplished through the miniaturization of one and two-dimensional gas chromatographs, preconcentrators, and detector components (IMS and PDHID). The system as configured currently is capable of meeting current and future challenges, covering the application spaces of both civilian and the military. 


\section{ACKNOWLEDGEMENTS}

This work was supported by the Defense Advanced Research Projects Agency Microsystems Technology Office (DARPA MTO) under Micro Gas Analyzers Program contract number 017040518. Sandia National Laboratories is a multimission laboratory managed and operated by National Technology \& Engineering Solutions of Sandia, LLC, a wholly owned subsidiary of Honeywell International Inc., for the U.S. Department of Energy's National Nuclear Security Administration under contract DE-NA0003525. This paper describes objective technical results and analysis. Any subjective views or opinions that might be expressed in the paper do not necessarily represent the views of the U.S. Department of Energy or the United States Government. The authors gratefully acknowledge consultations with Professor Richard Sacks at the University of Michigan, who inspired this avenue of research; and the assistance of Atilla Kiss and Akos Sule at Neptune Research. A part of this paper was supported by Sandia National Laboratories' Laboratory Directed Research and Development (LDRD) project \#s 199974.

\section{REFERENCES}

[1] Terry, S. C., Jerman, J. H. and Angell, J. B., "A gas chromatographic air analyzer fabricated on a silicon wafer," IEEE transactions on electron devices. 1880-1886 (1979).

[2] Matzke, C. M., Kottenstette, R. J., Casalnuovo, S. A., Frye-Mason, G. C., Hudson, M. L., Sasaki, D. Y., Manginell, R. P. and Wong, C. C., "Microfabricated silicon gas chromatographic microchannels: Fabrication and performance," Micromachining and Microfabrication Process Technology IV, 262-269 (1998).

[3] Heller, E., Hietala, V., Kottenstette, R., Manginell, R., Matzke, C., Lewis, P., Casalnuovo, S. and Frye-Mason, G., "An integrated surface acoustic wave-based chemical microsensor array for gas-phase chemical analysis microsystems," Chemical Sensors IV: Proceedings of the Symposium, 138 (1999).

[4] Manginell, R., Moorman, M., Achyuthan, K., Simonson, R. J., Mowry, C., Pfeifer, K., Pfeifer, N., Sillerud, C., Schwindt, P. and Carson, B., "Field detection of chemical agents and vocs as early symptomatic markers," https://www.osti.gov/servlets/purl/1426426 (2017)

[5] "'Nobel prizes of technology" demonstrate wide scope sandia researchers win seven r\&d 100 awards," https://shareng.sandia.gov/news/resources/releases/2003/other/rd1002003.html (2003)

[6] Manginell, R., Bauer, J., Moorman, M., Sanchez, L., Anderson, J., Whiting, J., Porter, D., Copic, D. and Achyuthan, K., "A monolithically-integrated $\mu \mathrm{gc}$ chemical sensor system," Sensors. 6517-6532 (2011).

[7] Manginell, R. P., Adkins, D. R., Moorman, M. W., Hadizadeh, R., Copic, D., Porter, D. A., Anderson, J. M., Hietala, V. M., Bryan, J. R. and Wheeler, D. R., "Mass-sensitive microfabricated chemical preconcentrator," Journal of Microelectromechanical Systems. 1396-1407 (2008).

[8] Whiting, J. J., Fix, C. S., Anderson, J. M., Staton, A. W., Manginell, R. P., Wheeler, D. R., Myers, E. B., Roukes, M. L. and Simonson, R., "High-speed two-dimensional gas chromatography using microfabricated gc columns combined with nanoelectromechanical mass sensors," TRANSDUCERS 2009-2009 International Solid-State Sensors, Actuators and Microsystems Conference, 1666-1669 (2009).

[9] Whiting, J. J., Myers, E., Manginell, R. P., Moorman, M. W., Anderson, J., Fix, C. S., Washburn, C., Staton, A., Porter, D., Graf, D., Wheeler, D. R., Howell, S., Richards, J., Monteith, H., Achyuthan, K. E., Roukes, M. and Simonson, R. J., "A high-speed, high-performance, microfabricated comprehensive two-dimensional gas chromatograph," Lab on a Chip. (2019).

[10] Eiceman, G. and Schmidt, H., [Aspects of explosives detection], "Advances in ion mobility spectrometry of explosives," Elsevier, 171-202 (2009)

[11] Pfeifer, K. B. and Rohde, S. B., "Signal-to-noise and resolution enhancement in ion mobility spectrometry using correlation gating techniques: Barker codes," IEEE Sensors Journal. 1130-1137 (2007).

[12] Manginell, R. P., Mowry, C. D., Pimentel, A. S., Mangan, M. A., Moorman, M. W., Sparks, E. S., Allen, A. and Achyuthan, K. E., "Development of a mesoscale pulsed discharge helium ionization detector for portable gas chromatography," Analytical Sciences. 1183-1188 (2015).

[13] Manginell, R. P., Pimentel, A. S., Mowry, C. D., Mangan, M. A., Moorman, M. W., Allen, A., Schares, E. S. and Achyuthan, K. E., "Diagnostic potential of the pulsed discharged helium ionization detector (pdhid) for pathogenic mycobacterial volatile biomarkers," Journal of breath research. 037107 (2013).

[14] Achyuthan, K., Harper, J., Manginell, R. and Moorman, M., "Volatile metabolites emission by in vivo microalgaean overlooked opportunity?," Metabolites. 39 (2017). 
[15] Brocato, T., Hess, R. F., Moorman, M. and Simonson, R. J., "Microfabricated nitrogen-phosphorus detector (unpd)determination of sensitivity and selectivity," Sandia National Lab.(SNL-NM), Albuquerque, NM (United States), SAND2015-2012J, (2013)

[16] Li, M., Myers, E., Tang, H., Aldridge, S., McCaig, H., Whiting, J., Simonson, R., Lewis, N. S. and Roukes, M., "Nanoelectromechanical resonator arrays for ultrafast, gas-phase chromatographic chemical analysis," Nano letters. 3899-3903 (2010). 